\title{
Umbilical cord mesenchymal stem cells-derived exosomes deliver miR-21 to promote corneal epithelial wound healing through PTEN/PI3K/Akt pathway
}

\section{Xiaolong Liu}

Northeast Agricultural University

Xuran Li

First Affiliated Hospital of Harbin Medical University

\section{Guangyuan Wu}

First Affiliated Hospital of Harbin Medical University

\section{Pengfei Qi}

First Affiliated Hospital of Harbin Medical University

\section{Yanyan Zhang}

First Affiliated Hospital of Harbin Medical University

Zhiyu Liu

First Affiliated Hospital of Harbin Medical University

\section{Xinyue Li}

First Affiliated Hospital of Harbin Medical University

Yu Yu

First Affiliated Hospital of Harbin Medical University

\section{Xiangmei Ye}

First Affiliated Hospital of Harbin Medical University

\section{Yang Li}

First Affiliated Hospital of Harbin Medical University

\section{Dongguang Yang}

First Affiliated Hospital of Harbin Medical University

\section{Yueqiu Teng}

First Affiliated Hospital of Harbin Medical University Ce Shi

First Affiliated Hospital of Harbin Medical University Xin Jin

First Affiliated Hospital of Harbin Medical University

Sen Qi

First Affiliated Hospital of Harbin Medical University 


\section{Yuting Liu}

First Affiliated Hospital of Harbin Medical University

\section{Shudan Wang}

First Affiliated Hospital of Harbin Medical University

\section{Ying Liu}

First Affiliated Hospital of Harbin Medical University

\section{Fenglin Cao}

First Affiliated Hospital of Harbin Medical University

\section{Qingran Kong}

Northeast Agricultural University

Zhenkun Wang ( $\nabla$ wzkcc@163.com )

First Affiliated Hospital of Harbin Medical University

\section{Hong Zhang}

First Affiliated Hospital of Harbin Medical University

\section{Research Article}

Keywords: Corneal wound healing, Umbilical cord mesenchymal stem cell, Exosomes, miR-21, PTEN

Posted Date: January 21st, 2022

DOI: https://doi.org/10.21203/rs.3.rs-571150/v2

License: (1) (i) This work is licensed under a Creative Commons Attribution 4.0 International License. Read Full License 
Umbilical cord mesenchymal stem cells-derived exosomes deliver miR-21 to promote corneal epithelial wound healing through PTEN/PI3K/Akt pathway

3

4 Xiaolong Liu ${ }^{1,4 \#}$, Xuran $\mathrm{Li}^{2,3 \#}$, Guangyuan $\mathrm{Wu}^{1,3}$, Pengfei Qi ${ }^{1,3}$, Yanyan Zhang ${ }^{2}$, Zhiyu Liu ${ }^{5}$, Xinyue

$5 \mathrm{Li}^{2}, \mathrm{Yu} \mathrm{Yu}^{2}$, Xiangmei $\mathrm{Ye}^{1,3}$, Yang $\mathrm{Li}^{1,3}$, Dongguang Yang ${ }^{1,3}$, Yueqiu Teng ${ }^{1,3}, \mathrm{Ce} \mathrm{Shi}^{1,3}, \mathrm{Xin}^{\mathrm{Jin}^{2}}$, Sen

$6 \mathrm{Qi}^{6}$, Yuting Liu ${ }^{2}$, Shudan Wang ${ }^{2}$, Ying $\mathrm{Liu}^{2}$,Fenglin Cao ${ }^{1,3}$, Qingran $\mathrm{Kong}^{4}$, Zhenkun Wang ${ }^{1,3 *}$ and

7 Hong Zhang ${ }^{2,3 *}$

$8 \quad{ }^{1}$ Central Laboratory, First Affiliated Hospital, Harbin Medical University, Harbin, China

$9 \quad{ }^{2}$ Eye Hospital, First Affiliated Hospital, Harbin Medical University, Harbin, China

${ }^{3}$ NHC Key Laboratory of Cell Transplantation, Harbin Medical University, Harbin, China

${ }^{4}$ College of Life Science, Northeast Agricultural University, Harbin, China

12

${ }^{5}$ Department of Laboratory Diagnostics, First Affiliated Hospital, Harbin Medical University,

Harbin, China

${ }^{6}$ Department of Hematology, First Affiliated Hospital, Harbin Medical University, Harbin, China

* Corresponding author.

Hong Zhang: zhanghong@hrbmu.edu.cn; Zhenkun wang:wzkcc@163.com

\# These authors contributed equally to this work.

\section{Abstract}

Objective: Rapid restoration of corneal epithelium integrity after injury is particularly important for preserving corneal transparency and vision. Mesenchymal stem cells (MSCs) can be taken into account as the promising regenerative therapeutics for improvement of wound healing processes 
based on the variety of the effective components. The extracellular vesicles form MSCs, especially exosomes, has been considered as important paracrine mediators though transferring microRNAs into recipient cell. This study investigated the mechanism of human umbilical cord MSC-derived exosomes (HUMSC-exosomes) on corneal epithelial wound healing.

Methods: Exosomes extracted from HUMSCs were identified by transmission electron microscopy, nanoparticle tracking analysis, and Western blot. Corneal fluorescein staining and histological staining were evaluated in a corneal mechanical wound model. Changes in HCECs proliferation after HUMSC-exosomes or miR-21 mimics treatment were evaluated by CCK-8 and EdU assays, while migration was assessed by in vitro scratch wound assay. Full-length transcriptome sequencing was performed to identify the differentially expressed genes associated with HUMSC-exosomes treatment, followed by validation via real-time PCR and Western blot.

Results: The exosomes derived from HUMSCs can significantly promote corneal epithelial cells proliferation, migration in vitro and corneal epithelial wound healing in vivo. Similar effects were obtained after miR-21 transfection, while the beneficial effects of HUMSC-exosomes were partially negated by miR-21 knockdown. Results also show that the benefits are associated with decreased PTEN level and activated the PI3K/Akt signaling pathway in HCECs.

Conclusion: HUMSC-exosomes could enhance the recovery of corneal epithelial wounds though restraining PTEN by transferring miR-21, and may represent a promising novel therapeutic agent for corneal wound repair.

Keywords: Corneal wound healing; Umbilical cord mesenchymal stem cell; Exosomes; miR-21; PTEN 


\section{Introduction}

47 Superficial corneal lesions can heal rapidly and without complication, however, delayed corneal

48

49 epithelial healing can lead to subsequent corneal infections with further complications, such as corneal scarring, thinning, ulceration and even perforation. According to the World Health Organization, it is estimated that corneal opacities, including corneal ulceration, are the fourth leading cause of blindness worldwide [1]. Although several therapies exist and an increasing number of novel approaches are emerging, treatment of severe corneal epithelial defect can still be quite challenging. Therefore, a topical treatment that aids in the management and accelerated closure of corneal wounds would help reduce the risk of infections and scarring, and thus improve visual outcomes.

Mesenchymal stem cells (MSCs)-based therapies participated in renovating the structure and function of damaged or diseased tissues[2]. However, poor engraftment and limited differentiation of transplanted MSCs suggest that their beneficial effects might not be associated with their differentiation and direct replenishment of damaged tissue parenchymal cells [3, 4]. Currently, emerging evidence has shown that the therapeutic effect of MSCs mainly relies on paracrine activities [5]. MSCs can release extracellular vesicles (EVs) containing bioactive molecules that affect cellular processes in neighboring cells. Therefore, it may be possible to avoid the limitations and complications of stem cell therapy in the eye by using MSC derived EVs as biomimetic agents to accelerate corneal wound healing [6]. 
EVs, specifically exosomes, are functional paracrine units of stem cells and have therapeutic effects similar to their parent cells, suggesting that they may provide a promising, cellfree therapeutic options [7-9]. The application of MSC-derived exosomes alone can exert similar functions of MSCs and participate in the regulation of immune response, inflammatory disease and wound healing [10]. The cellular bilayer lipid membrane of exosomes protected proteins, mRNAs, and non-coding RNAs (ncRNAs) from destruction, which can be transferred to recipient cells for cell-to-cell communication [11]. ncRNAs is considered as key post-transcriptional modulators of gene expression and can be transferred in active form via exosomes to regulate the activity of certain cells. Among them, microRNAs (miRNAs) have emerged as the most important modulator [12]. miRNAs are a class of evolutionally conserved, single-stranded ncRNAs, which are either transcribed by RNA polymerase II from independent genes or introns of protein-coding genes [13]. miRNAs are crucial players during normal development, homeostasis, and disease, which participate in almost every biological process such as cell proliferation and survival [14].

MSCs from cord tissues are easily attainable and more primitive than MSCs isolated from adult sources. Previous studies have shown that human umbilical cord mesenchymal stem cell derived exosomes (HUMSC-exosomes) could transfer miRNAs and attenuate cell death and enhance cutaneous wound healing $[15,16]$.Considering the similar wound healing process between skin and corneal, we studied the functions of HUMSC-exosomes in corneal wound repair. The present study demonstrated the therapeutic effect of HUMSC-exosomes using a corneal mechanical wound model. To investigate the mechanism underlying HUMSC-exosomes-mediated corneal wound repair, we studied the effects of HUMSC-exosomes on human corneal epithelial cells (HCECs) migration and 
proliferation. Through high-throughput sequencing and bioinformatics analysis, we identified that miR-21 are carried by HUMSC-exosomes as crucial elements contributing to HCECs migration and proliferation by down-regulating PTEN expression.

\section{Materials and Methods}

\section{Primary cell culture and Characterization}

The umbilical cord was obtained from the Department of Obstetrics and Gynecology of the First Affiliated Hospital of Harbin Medical University after harvesting informed consent for research purposes, which was approved by the Ethics Committee (ethical approval number: 201859). The collected umbilical cord Wharton's jelly tissue was cut into small pieces, and then allowed to stick to the bottom of the cell culture plates. Dulbecco's Modified Eagle's Medium (DMEM) Low Glucose with $10 \%$ fetal bovine serum (FBS) and $100 \mathrm{U} / \mathrm{mL}$ penicillin-streptomycin (Gibco, USA) were added to the cells. The dissociated cells were washed with PBS, and stained with antibodies CD90, CD105, CD73, CD34, CD11b, CD19, CD45 and HLA-DR using the BD ${ }^{\mathrm{TM}}$ Human MSC Analysis Kit. The FACS analysis was performed using a FACS Calibur ${ }^{\mathrm{TM}}$ flow cytometer (BD Biosciences), and the data were analyzed using FlowJo software (BD Biosciences). The release of EVs from HUMSCs was blocked by pre-incubating HUMSCs with $20 \mu \mathrm{M}$ GW4869 (sigma, USA) for 24 hours. All cells used in our experiments were from early passages 3 to 5 .

To avoid the influence of FBS-derived exosomes on HUMSC-exosomes, HUMSCs used for exosomes extraction were cultured using exosomes-free FBS which were centrifuged at 120,000 g at $4{ }^{\circ} \mathrm{C}$ for 18 hours using a Beckman Optima L-100 XP ultracentrifuge with a SW 32 Ti rotor. 
HUMSCs supernatants was collected at different times every 24-48 hours and centrifuged at $300 \mathrm{~g}$ $120,000 \mathrm{~g}$ for another 70 minutes. All centrifugation steps were performed at $4^{\circ} \mathrm{C}$. The purified exosomes were resuspended in $\mathrm{PBS}$ and stored at $-80^{\circ} \mathrm{C}$.

The concentration of HUMSC-exosomes was determined by BCA protein assay kit, as suggested by the manufacturer (Beyotime Institute of Biotechnology, China). The morphology of HUMSCexosomes was observed by transmission electron microscopy (TEM) (JEOL JEM-1220, Japan). And the size distribution of HUMSC-exosomes was measured by nanoparticle tracking analysis (NTA, Malvern Zetasizer, England). The membrane protein marker (CD9, CD81, CD63) were analyzed using Western blot. To obtain the miR-21 knockdown HUMSC-exosomes, we transfected MSCs with miR-21 inhibitors (RIBOBIO, China) or negative control (NC) using Opti-MEM (Gibco, USA) and Lipofectamine 2000 (Invitrogen) according to the manufacturer's instructions. After 48 hours 
133

\section{HCECs culture and transfection}

The human corneal epithelial cell line (HCEC, Bnbio, China) were cultured in DMEM High Glucose supplemented with 10\% FBS (Gibco, USA). HCECs were seeded into 6-well or 12-well plates the day before treatment. Prior to HUMSC-exosomes or PBS treatment, HCECs were starved in serum-free DMEM for 24 hours at 50\% confluence. HCECs were transfected with miR-21 mimics or miR-21 inhibitors and corresponding NC, pCDNA3.1-3 $\times$ Flag-PTEN and empty vector plasmid as indicated.

\section{Exosome uptake assay}

For the exosome uptake analysis, $20 \mu \mathrm{g}$ purified HUMSC-exosomes in $100 \mu \mathrm{l}$ PBS was incubated with $1 \mu \mathrm{M}$ Dil (Beyotime Institute of Biotechnology, China) in the dark for 30 min, washed twice with PBS, ultracentrifuged at $120,000 \mathrm{~g}$ for $70 \mathrm{~min}$ to remove nonbinding dye, and then resuspended in serum-free medium. HCECs were labeled with $5 \mathrm{mM} \mathrm{CFSE} \mathrm{in} \mathrm{the} \mathrm{dark} \mathrm{for} 20 \mathrm{~min}$, washed twice with complete medium. $200 \mu \mathrm{l}$ cell suspension $\left(5 \times 10^{4} / \mathrm{ml}\right)$ was seeded into $35 \mathrm{~mm}$ glass bottom dishes (Cellvis, USA) and let the cells adhere to the glass for 12 hours. CFSE-labeled HCECs were co-cultured with Dil-labeled HUMSC-exosomes for 2 hours. After washed with PBS and fixed in 4\% paraformaldehyde, cell nuclei were stained with DAPI (Beyotime Institute of Biotechnology, China). Images were taken under confocal microscope (Zeiss LSM 710, Germany) and analyzed with supplementary software.

For TEM observation, HCECs were co-cultured with exosomes $(40 \mu \mathrm{g} / \mathrm{ml})$ for 2 hours and then fixed with $2.5 \%$ glutaraldehyde and postfixed with 3\% osmium tetroxide (OsO4) for 2 hours. The 
155

specimen was dehydrated in a graded series of ethanol, embedded in Epon resin and then imaged with TEM at $100 \mathrm{kV}$ (Hitachi H-7650, Japan).

\section{In vitro Wound healing assay}

HCECs were seeded into six-well plates and grown to confluence. The monolayer was scratched using a $200 \mu 1$ pipette tip and washed with serum-free medium to remove detached cells. Then, the cells were kept in co-culture with HUMSC-exosomes or not. At different times, images of wound scratch were taken under a microscope. The scratch closure was analyzed by ImageJ software. The percentage of wound closure was calculated as follows: migration area $(\%)=\left(A_{0}-A_{n}\right) / A_{0} \times 100$, where $A_{0}$ represents the initial wound area, and $A_{n}$ represents the wound area at the time of measurement.

\section{In vitro Cell proliferation assay}

HCECs proliferation was measured using the cell counting kit-8 (CCK-8, Sigma, USA) according to the manufacturer's protocol. The optical density (OD) at $450 \mathrm{~nm}$ was measured with averages from three replicates using a microplate reader (BioTek Instruments, USA).

\section{In vitro EdU proliferation assay}

Cell proliferation was also assessed using EdU Cell Proliferation Assay kit (RiboBio, China) according to the manufacturer's protocol. Briefly, after treatment, HCECs were exposed to $50 \mu \mathrm{M}$ 5-ethynyl-2'-deoxyuridine (EdU, RiboBio) for $2 \mathrm{~h}$ at $37^{\circ} \mathrm{C}$, and then the cells were fixed in $4 \%$ paraformaldehyde. After permeabilization with $0.5 \%$ Triton-X100, the cells were reacted with $1 \times$ 
177 Apollo reaction cocktail for 30 minutes. Subsequently, the DNA contents of the cells were stained 178 with Hoechst33342 for 30 minutes. Finally, the proportion of the cells incorporating EdU was 179 determined with fluorescence microscopy (OLYMPUS, IX51).

\section{Western blot}

HUMSC-exosomes and HCECs were lysed in lysis buffer containing protease and phosphatase inhibitor (Beyotime Institute of Biotechnology, China). Proteins was separated by electrophoresis after loading onto polyacrylamide gel, and then transferred to the PVDF that was incubated with primary antibodies against phospho-Akt (4060s, CST), PTEN (9188, CST), CD9 (ab92726, Abcam), CD61 (ab59479, Abcam), CD81 (00679767, Invitrogen) overnight at $4{ }^{\circ} \mathrm{C}$ after blocking with $5 \%$ nonfat milk, followed by incubation with horseradish peroxidase (HRP)-conjugated secondary antibody. Proteins were detected with a Western blot analysis system.

Total RNA of cells was extracted using TRIzol kit (Invitrogen, USA). RT-qPCR was carried out using the SYBR ${ }^{\circledR}$ Premix Ex TaqTM kit (Takara Bio, Japan) according to the manufacturer's instructions. The thermocycling conditions (Bio-Rad, CFX96) used were as follows: $95^{\circ} \mathrm{C}$ for 3 minutes; followed by 40 cycles of $95^{\circ} \mathrm{C}$ for 5 seconds, $60^{\circ} \mathrm{C}$ for 30 seconds and $72^{\circ} \mathrm{C}$ for 30 seconds, calculated by the $2^{-\Delta \Delta \mathrm{Ct}}$ method. 
199

200

201

202

203

204

205

206

207

208

209

210

211

212

213

214

215

HUMSC-exosomes microRNA expression microarray GSE69909 were downloaded from GEO database. Target Scan, mirBase and miRDB were used to predict the target genes of miRNAs enriched in exosomes. All the predicted targets have target prediction scores $\geq 80$ were subjected to gene ontology (GO) analysis to investigate the underlying mechanism of the potential HUMSCexosomes miRNA and the target mRNAs during corneal re-epithelialization.

\section{Full-length transcriptome sequencing}

HCECs $\left(2.5 \times 10^{6}\right.$ cells $)$ were treated with $40 \mu \mathrm{g} / \mathrm{ml}$ HUMSC- exosomes for 48 hours, same volume of PBS was added as control with three biological replicates. Total RNA was isolated using the TRIzol reagent according to the manufacturer's instructions. $1 \mu \mathrm{g}$ total RNA was prepared for cDNA libraries using cDNA-PCR Sequencing Kit (SQK-PCS109) protocol provided by Oxford Nanopore Technologies (ONT). Briefly, the template switching activity of reverse transcriptase enrich for full-length cDNAs and add defined PCR adapters directly to both ends of the first-strand cDNA. And following cDNA PCR for 14 circles with LongAmp Tag (NEB). The PCR products were then subjected to ONT adaptor ligation using T4 DNA ligase (NEB). Agencourt XP beads was used for DNA purification according to ONT protocol. The final cDNA libraries were added to FLOMIN109 flowcells and run on PromethION platform at Biomarker Technology Company (Beijing, China). KOBAS software was used to test the statistical enrichment of differential expression transcripts in KEGG pathways.

\section{Corneal mechanical wound model and treatment}

The experimental protocols were approved by The Ethics Committee of First Affiliated Hospital of 
221

222

223

224

Harbin Medical University (ethical approval number: 2020100). Weight from 160-180g male Sprague-Dawley rats (6-8 weeks old) were purchased from the animal experiment center of the Second Affiliated Hospital of Harbin Medical University. The rats were anesthetized with intraperitoneal injection and applied topically $0.5 \%$ proparacaine, the corneal epithelium was removed up to the corneal/limbal border with AlgerBrush II (The Alger Company, Lago Vista, TX, USA) as previously described [17]. A unilateral corneal injury was created. Protocols were approved by the Harbin Medical University Animal Care and Use Committee guideline.

Rats were randomly divided into four groups and subconjunctival injected with $100 \mu$ PBS containing $2 \times 10^{6}$ HUMSCs, equal amount of HUMSCs pretreated with GW4869, $40 \mu \mathrm{g}$ HUMSCexosomes or an equal volume of PBS, respectively. Wound residual area was monitored every 12 hours using fluorescein staining and photographed using a camera equipped Nikon FS-2 slit lamp biomicroscope. The percentages of residual defect were analyzed by ImageJ software.

\section{Histological analysis}

The eyes were enucleated and post-fixed with 4\% paraformaldehyde within 10 minutes after euthanasia. $4 \mu \mathrm{m}$ paraffin-embedded sections stained with hematoxylin and eosin (H\&E) were used to observe the corneal structure and degree of corneal re-epithelialization. The sections were photographed under light microscope (Olympus, Japan).

\section{Statistical analysis}

All statistical analyses were performed using Prism software. Data are summarized as mean \pm 
243

standard deviation (SD). Student $t$-test was used to determine statistically significant differences between samples. When multiple comparison analyses were required, statistical significance was evaluated by one-way ANOVA. All $P$-values $<0.05$ were considered statistically significant.

\section{Results}

\section{Identification of HUMSCs and HUMSC-exosomes}

Results of flow cytometry analysis confirmed the presence of positive expressions of typical MSC makers CD105, CD90, CD73, while the surface markers of hematopoietic cells such as CD34, CD11b, CD19, CD45 and HLA-DR were fairly weak to detect compared with the isotype control (Fig. 1(a)). In addition, according to inverted microscopic observation, the morphology of the cells was regular long spindle with directional arrangement, and presented a typical spindle shape, which grew as whirlpool or cluster (Fig. 1(b)).

The classical structure of the isolated exosome, including "rim of a cup" and double-layer membrane morphology, were observed by TEM (Fig. 1(c)). NTA results demonstrated that the diameters of the particles were around 50-150 nm (Fig. 1(d)). The identity of these particles was further confirmed as exosomes by Western blot, which showed the presence of widely expressed exosomal markers, including CD9, CD63, CD81 (Fig. 1(e)). Therefore, results above confirmed that the EVs we extracted were indeed exosomes. 
A
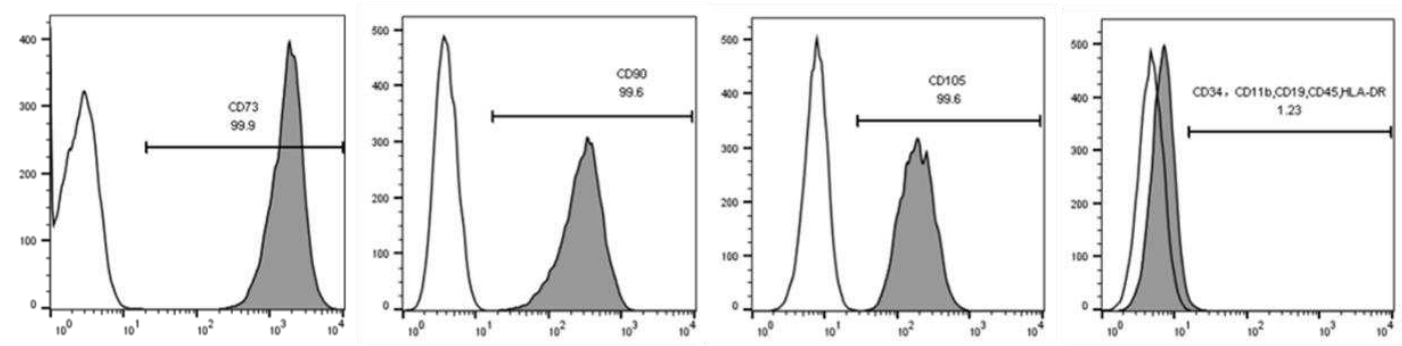

B

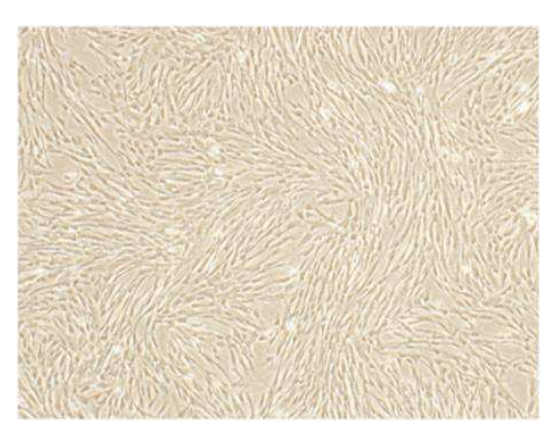

D

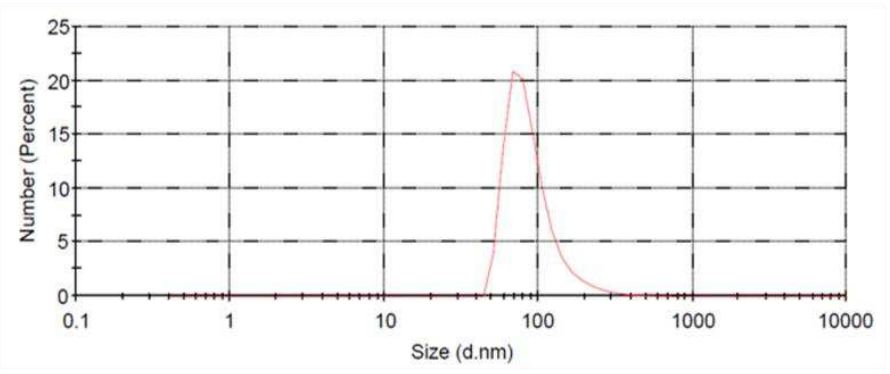

C

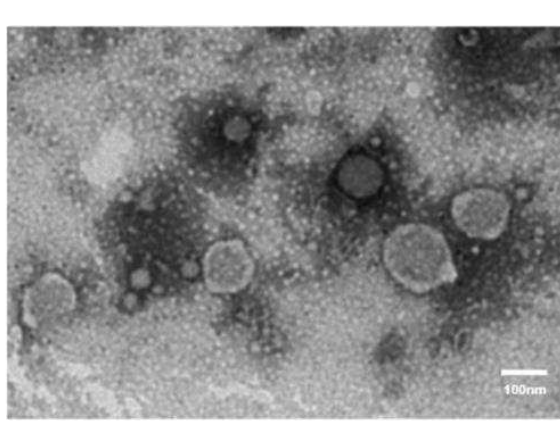

E

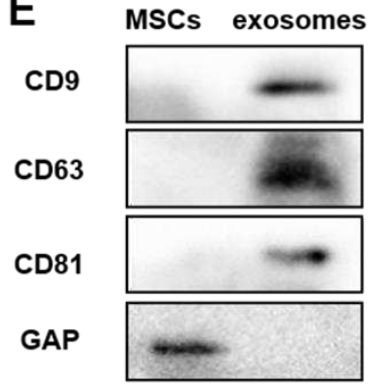

Figure. 1

Morphological observation and identification of HUMSCs and HUMSC-exosomes. (a) Flow cytometry analysis of surface markers in HUMSCs. (b) Light morphology image of HUMSCs. (c) Morphology of HUMSC-exosomes under TEM. Scale bar, $100 \mathrm{~nm}$. (d) Peak size of HUMSC-exosomes was around $80 \mathrm{~nm}$ as showed by NTA. (e) HUMSC-exosomes were positive for CD9, CD81 and CD63 as indicated by Western blot.

2. Application of HUMSCs or HUMSC-exosomes promotes corneal wound healing in a rat model

We found that subconjunctival injection of HUMSCs or HUMSC-exosomes can effectively promote the healing of corneal defects in rats, while inhibition of exosome secretion by GW4869 can 
273 attenuated HUMSCs mediated benefits at 24 hours (Fig. 2(a) and (c)). The injured corneas

274 treated with HUMSC and HUMSC-exosomes regained more regular arrangement and

275 compact structure than those treated with PBS through assessing corneal tissues microstructure by

276 H\&E staining (Fig. 2(b)). 

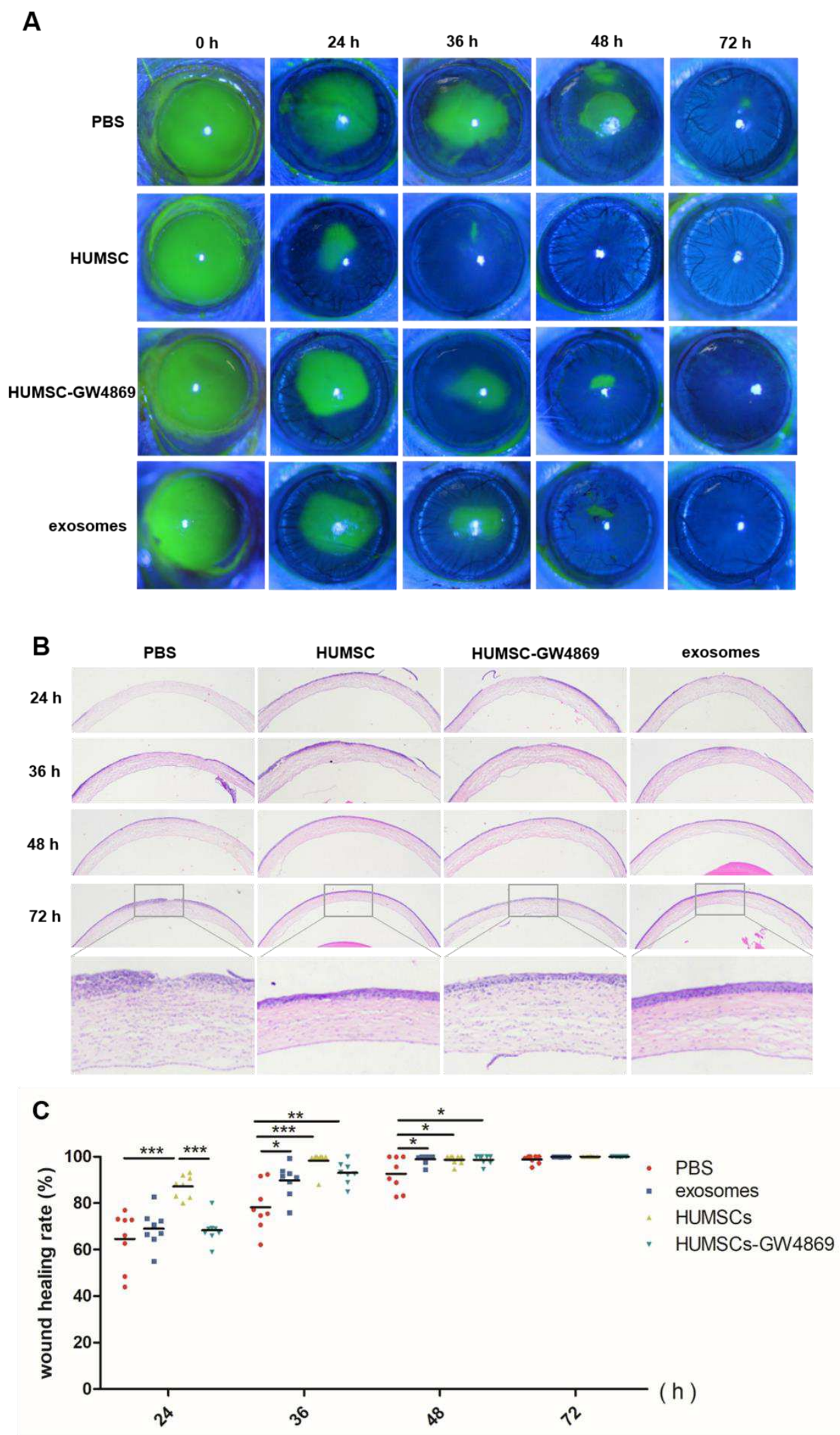

Figgure. 2

279 The effect of HUMSCs and HUMSC-exosomes on corneal epithelial wound healing in vivo. (a, c) Fluorescein- 
stained images of defect corneas, before and after treatment with HUMSCs, HUMSCs-GW4869, HUMSC-exosomes

or PBS. (b) H\&E staining showed the histologic appearance of the cornea. Data are expressed as the means $\pm \mathrm{SD}$.

${ }^{*} P<0.05,{ }^{* *} P<0.01$, and $* * * P<0.001, n=8$.

\section{HUMSC-exosomes promote the proliferation and migration of HCECs in vitro}

To demonstrate the uptake of exosomes, CFSE-labelled HCECs were co-cultured with Dil-labelled exosomes and then visualized with laser scanning confocal microscope. The red nanoparticles represent the labeled exosomes that occurred in smaller clusters and were observed either surrounding the cell membrane or within the cytoplasm in HCECs. Localization results showed that exosomes derived from HUMSCs had been taken up by HCECs with the dye distributing within in the cell (Fig. 3(a)). In addition, the fusion process was also observed by TEM (Fig. 3(b)).

In order to evaluate whether HUMSC-exosomes stimulates HCECs migration, the effect on wound closure rates were investigated. The disparity of the remaining area during scratch wound assays confirmed the promigratory effects of HUMSC-exosomes with a dose-related trend after 18 hours incubation (Fig. 3(c) and (d)). Considering corneal healing is a dynamic interwoven process composed of cell proliferation, migration and adhesion, and the proliferation ability is the basis. We further investigated whether HUMSC-exosomes could enhance the proliferation-promoting behavior of HCECs in vitro. The CCK-8 assay showed that the proliferation of HCECs after incubating with exosomes was significantly improved in a dose-dependent manner (Fig. 3(e)). And the EdU assays for visualization of proliferating cells also demonstrated that HUMSC-exosomes treatment increased the percentage of proliferating cells compared to controls (Fig. 3(f) and (g)). 
A

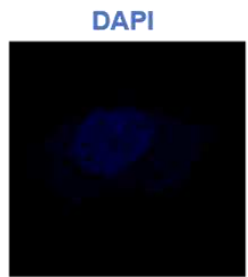

CFSE

DIL

Merge

B
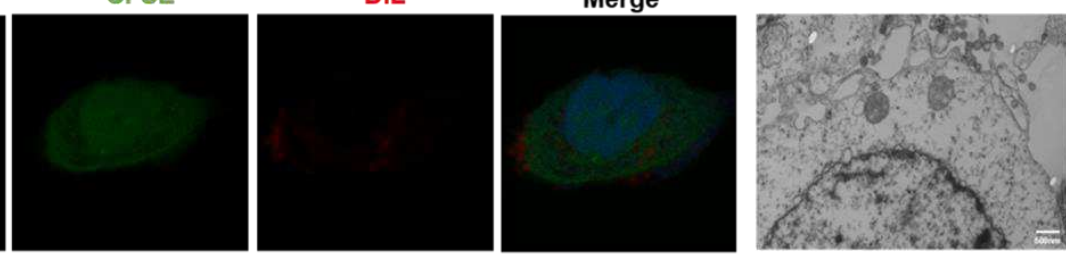

C

Oh
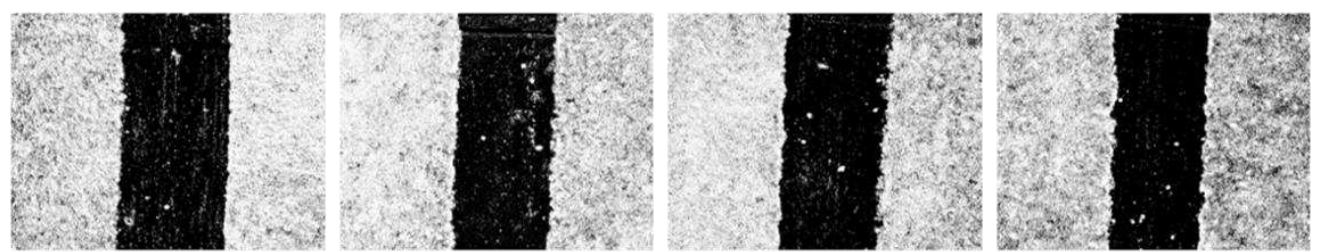

24h

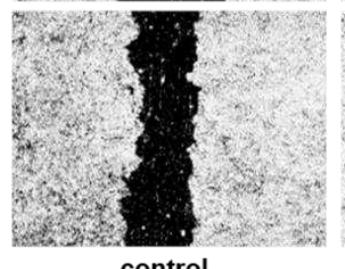

control
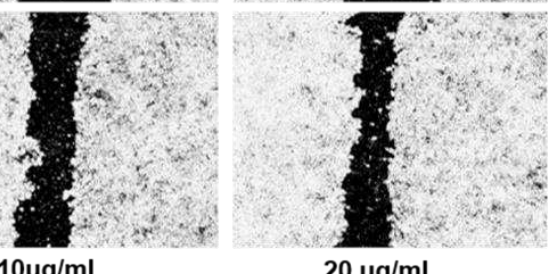

$20 \mu \mathrm{g} / \mathrm{ml}$

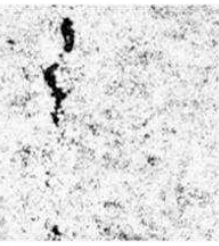

$40 \mu \mathrm{g} / \mathrm{ml}$

D

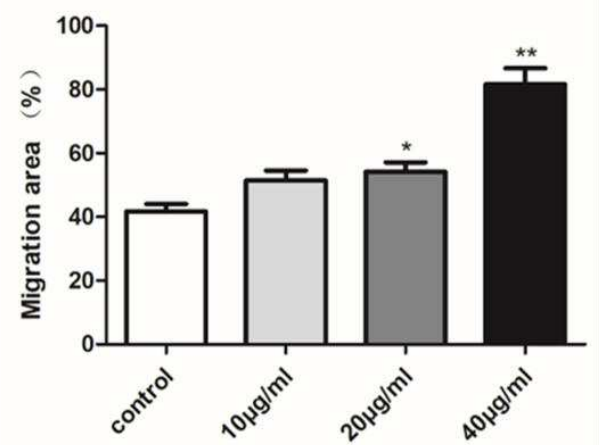

E

F
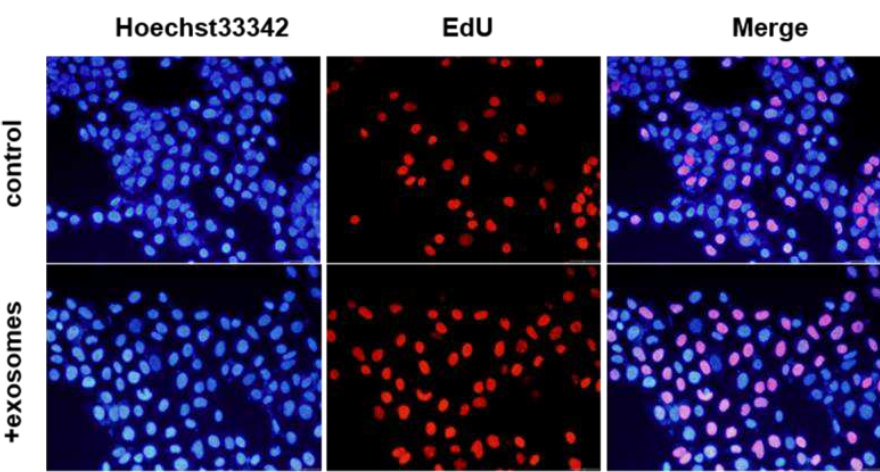

G
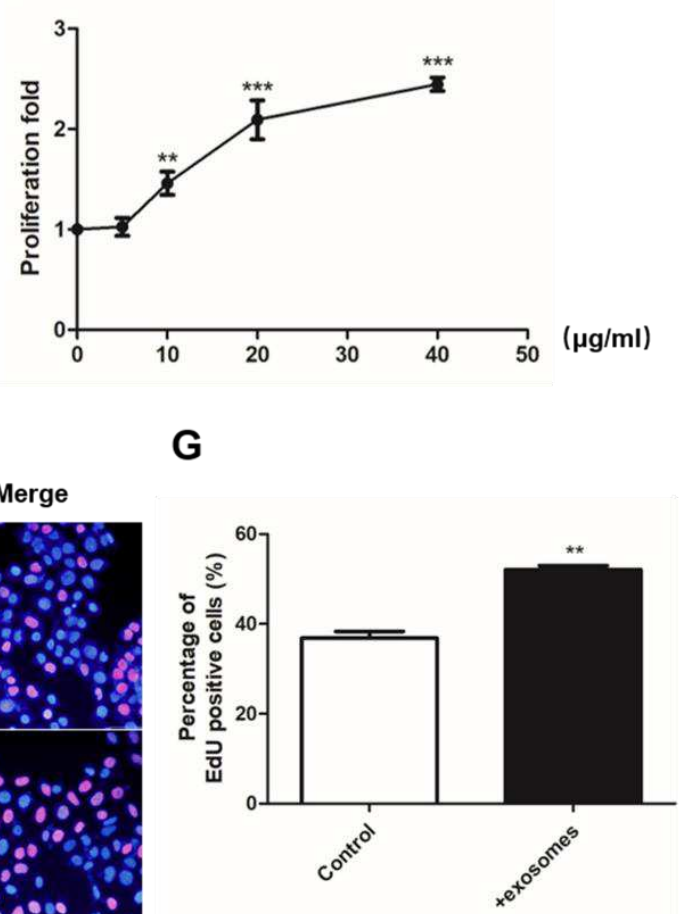

302 Figgure. 3

The effect of HUMSC-exosomes on HCECs proliferation and migration in vitro. (a) Fluorescence images

of CFSE-labelled HCECs (green) incubated with Dil-labelled HUMSC-exosomes (red). Nuclei were stained with 
the presence of HUMSC-exosomes when compared to controls, $n=4$. (e) CCK-8 assay showed increased proliferation of HCECs incubated with HUMSC-exosomes after 48 hours, $n=5$. (f, g) The proliferating HCECs was detected by EdU incorporation. The cells were treated with HUMSC-exosomes or blank control, $n=3$. Blue: nuclear staining (Hoechst33342); Red: EdU staining. Data are expressed as the means \pm SD. ${ }^{*} P<0.05$, $* * P<0.01$, and $* * * P<0.001$

\section{HUMSC-exosomes promote HCECs proliferation and migration though PI3K/Akt pathway}

To further investigate the potential mechanism of HUMSC-exosomes regulated proliferation and migration in HCECs, full-length transcriptome sequencing was used to detect the mRNA expression levels of related genes. 240 differentially expressed genes (DEG) were identified (Fold Change $\geq 2$ and $P$ value $<0.05$ ), including 104 up-regulated DEGs and 136 down-regulated DEGs (Fig. 4(a)). Then, we interpreted the potential biological functions of DEGs from the gene function and signaling pathway through KEGG enrichment analysis, and revealed that the PI3K/Akt signaling pathway, the phosphatidylinositol signaling system, cell adhesion molecules and MAPK signaling pathway had a significant difference between before and after HUMSC-exosome treated HCECs (Fig. 4(b)). Previous studies demonstrated that PI3K/Akt pathway involved deeply in the modulation of the process of corneal epithelial wound healing [18]. Therefore, the PI3K/Akt signaling pathways involved in HCECs proliferation and migration process after HUMSCexosomes treatment were explored. 
A

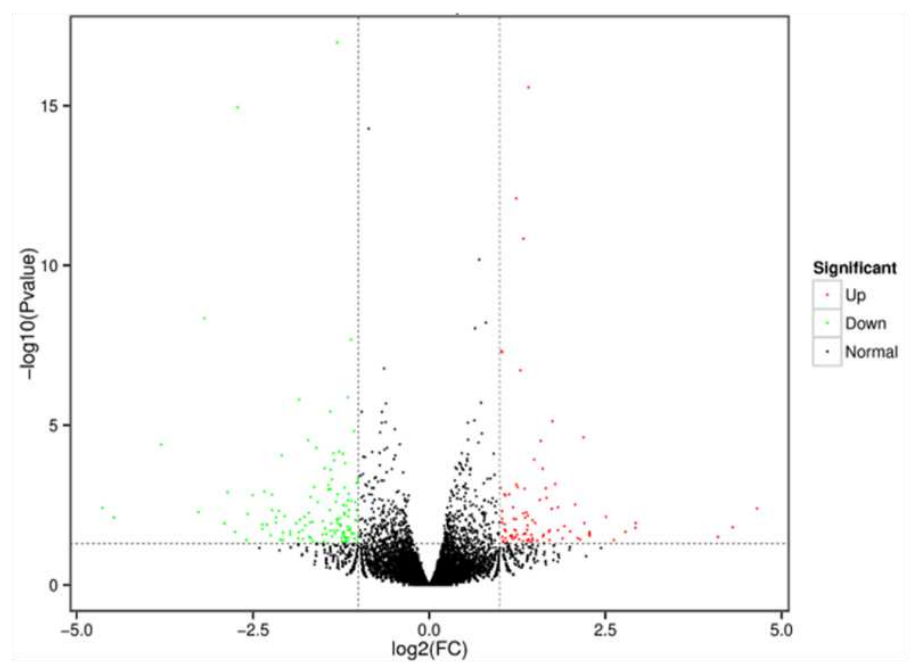

B

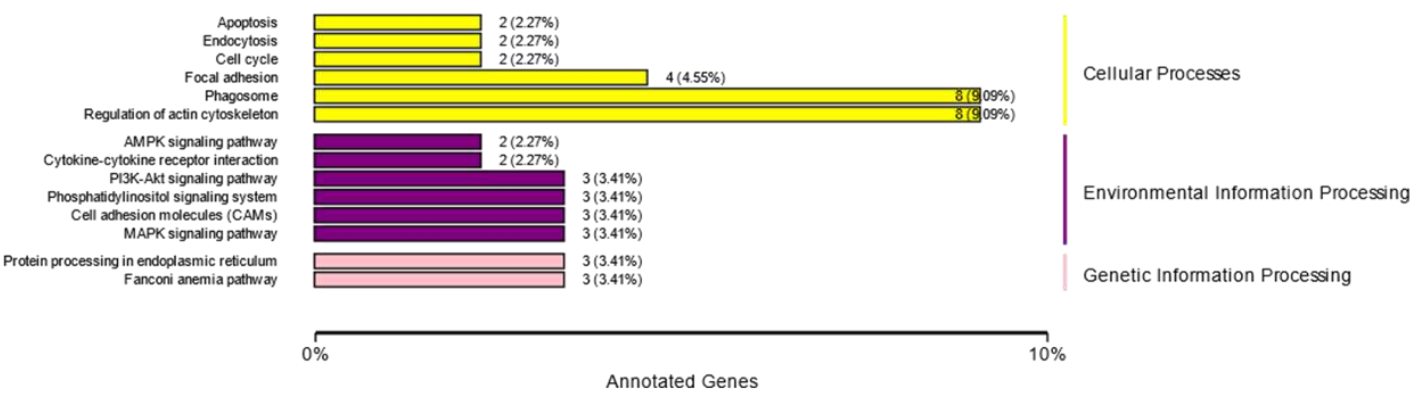

Figgure. 4

Transcriptome and pathway analysis of HUMSC-exosomes treatment. (a) Volcano Plot of DEGs between HUMSC-

regulated DEGs, black dots are non-significant DEGs. (b) The KEGG annotation results of the DEGs were classified according to the pathway types in KEGG. DEG, differentially expressed genes; KEGG, Kyoto Encyclopedia of

Genes and Genomes

\section{Exosomal miR-21 regulates the PTEN/ PI3K/AKT signaling pathway}


was used to determine the content of various miRNAs in HUMSC-exosomes [21]. Among the several miRNAs selectively enriched in HUMSC-exosomes, we focused on the most abundant one, miR-21(Fig. 5(a)). The downstream targets were predicted by Target Scan, mirBase and miRDB database, then imputed DAVID online to conduct GO analysis. The results showed that miR-21 was involved in the regulation of various molecular function, containing calcium ion binding, peptidase inhibitor activity, growth factor activity etc. (Fig. 5(b)). Among them, the phosphatase activity may involve in the regulation of PI3K/Akt. miRNAs can exert their functions by interacting with the $3^{\prime}$ untranslated region (3' UTR) or protein coding sequence of target mRNAs. According to the miRbase database, PTEN might be the potential downstream of miR-21 (Fig. 5(c)).

A

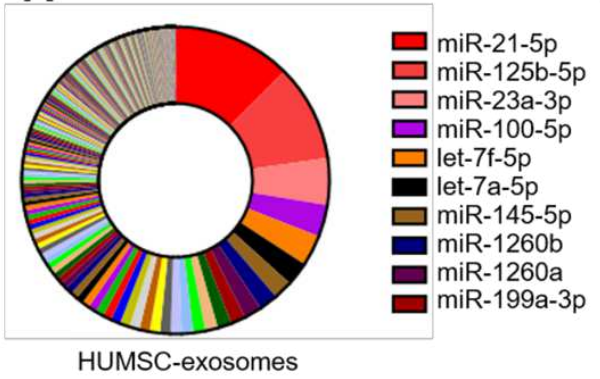

B

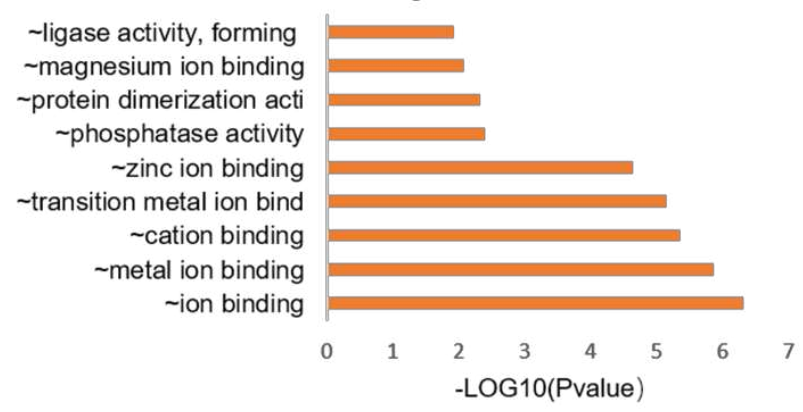

C PTEN-3'UTR 5' CUGGUUCUAAGCUAG miR-21 3' UCAGACUAUUCGAU

Figgure. 5

Identification of HUMSC-exosomal microRNAs. (a) miRNAs abundance analysis of HUMSC-exosomes. (b) mRNA targets for the microRNAs significantly enriched in HUMSC-exosomes were identified and GO analysis. (c)

The binding site between miR-21 and PTEN mRNA. MF, molecular function; GO, Gene Ontology

Unsurprisingly, we found that reduced mRNA and protein expression levels of PTEN were identified within HCECs after treated with HUMSC-exosomes (Fig. 6(a) and (d)). The activation of 
the PI3K/Akt pathway in HCECs following HUMSC-exosome stimulation was verified by assessing the phosphorylated Akt levels (Fig. 6(d)). To confirm whether PTEN is a target of miR21 in HCECs, we further measured the expression of PTEN in HCECs transfected independently with miR-21 mimics or inhibitors and their corresponding NC to verify the interaction between the miR-21 and PTEN by qRT-PCR and Western blot. Once transfected with miR-21 mimics, the protein levels of PTEN were significantly reduced in HCECs (Fig. 6(f)), and the difference was also

362 detected in transcription level (Fig. 6(b) and (c)). We also found that the effects of miR-21 on the Akt phosphorylation were stimulative (Fig. 6(g)). Meanwhile, overexpression of PTEN downregulated the phosphorylated Akt levels, which was important for proliferation and migration (Fig. 6(e)). These results suggested that miR-21 regulate PTEN within HCECs via post-transcriptional modify. 
A

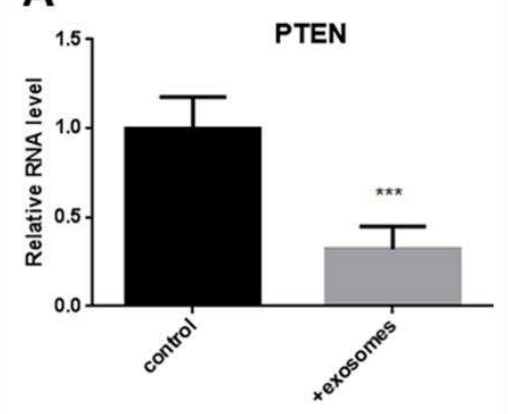

D

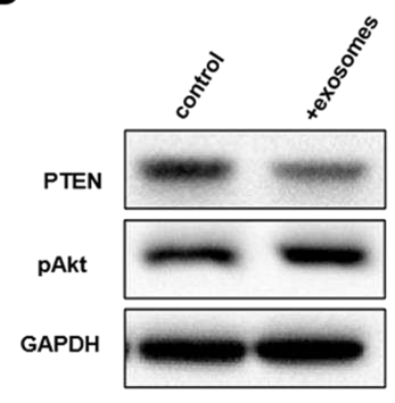

B

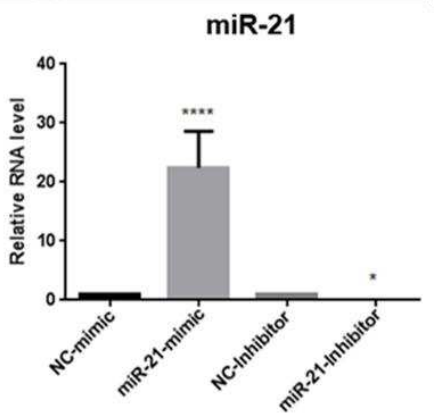

C

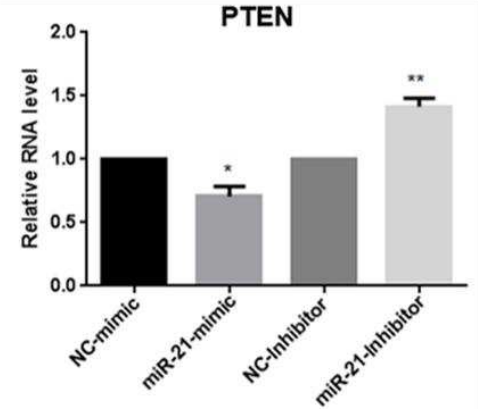

E

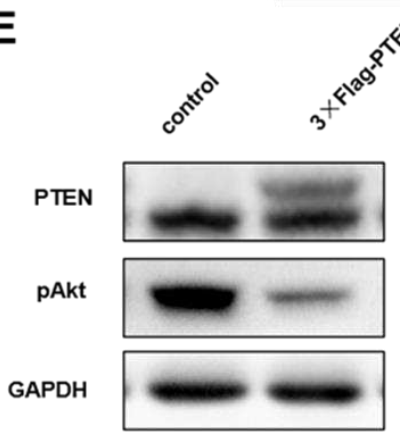

G

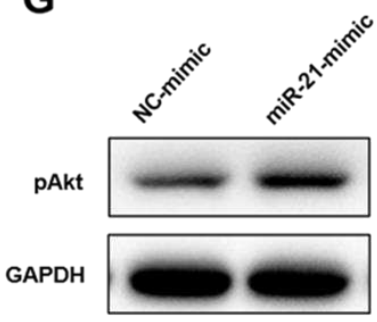

F

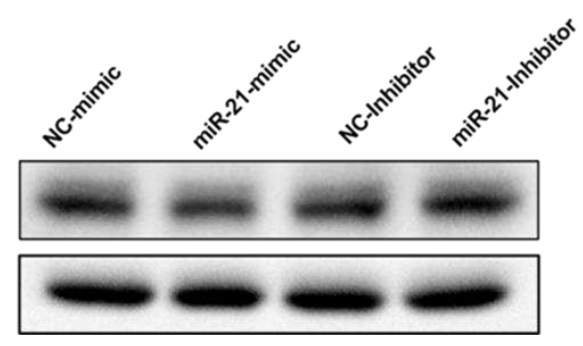

\section{HUMSC-exosomes promote HCECs proliferation and migration through miR-21}

Fgure. 6

Exosomal miR-21 regulate HCECs proliferation and migration by activating PI3K/Akt pathway through targeting PTEN. (a, d) HUMSC-exosomes treatment decreased the RNA and protein levels of PTEN in HCECs. (b) The expression level of miR-21 in HCECs. (c, f) The PTEN changed with miR-21 variation. (e) The expression of phospho-Akt after overexpression of PTEN. (g) The expression level of phospho-Akt after transfected with miR-21 mimics was detected by Western blot. Data are expressed as the means \pm SD. $* P<0.05, * * P<0.01$, and In order to assess whether the exosome-mediated miR-21 transfer plays a role in HCECs 
378

proliferation and migration, a subsequent knockdown experiment was conducted. HUMSCs were transfected with miR-21 inhibitors (at final concentration of $100 \mathrm{nM}$ ) or $\mathrm{NC}$, and the culture supernatants were collected subsequently for isolating the exosomes. Then, HCECs were incubated with the same concentration of miR-21 contained or miR-21 knockdown HUMSC-exosomes for migration and CCK-8 analysis. Results showed that the up-regulation of migration (Fig. 7(a) and (b)), as well as proliferation (Fig. 7(c)) induced by HUMSC-exosomes were partially negated by miR-21 knockdown.

To further study the potential involvement of miR-21, HCECs were transiently transfected with miR-21 mimics or NC. Proliferation of HCECs following transfection with miR-21 mimics or NC was assessed using CCK-8 and EdU assay. miR-21 mimics transfection significantly promoted the proliferation of HCECs compared with the NC group (Fig. 7(d)-(f)). In addition, the ability of HCECs transfected with miR-21 mimics to regain monolayer integrity was raise compared with NC-transfected cells (Fig. 7(g) and (h)).

Taken together, our data indicate that exosomal miR-21 promotes proliferation and migration by activating PI3K/Akt signaling pathway, which might play a critical role to enhance corneal epithelial wound healing. 

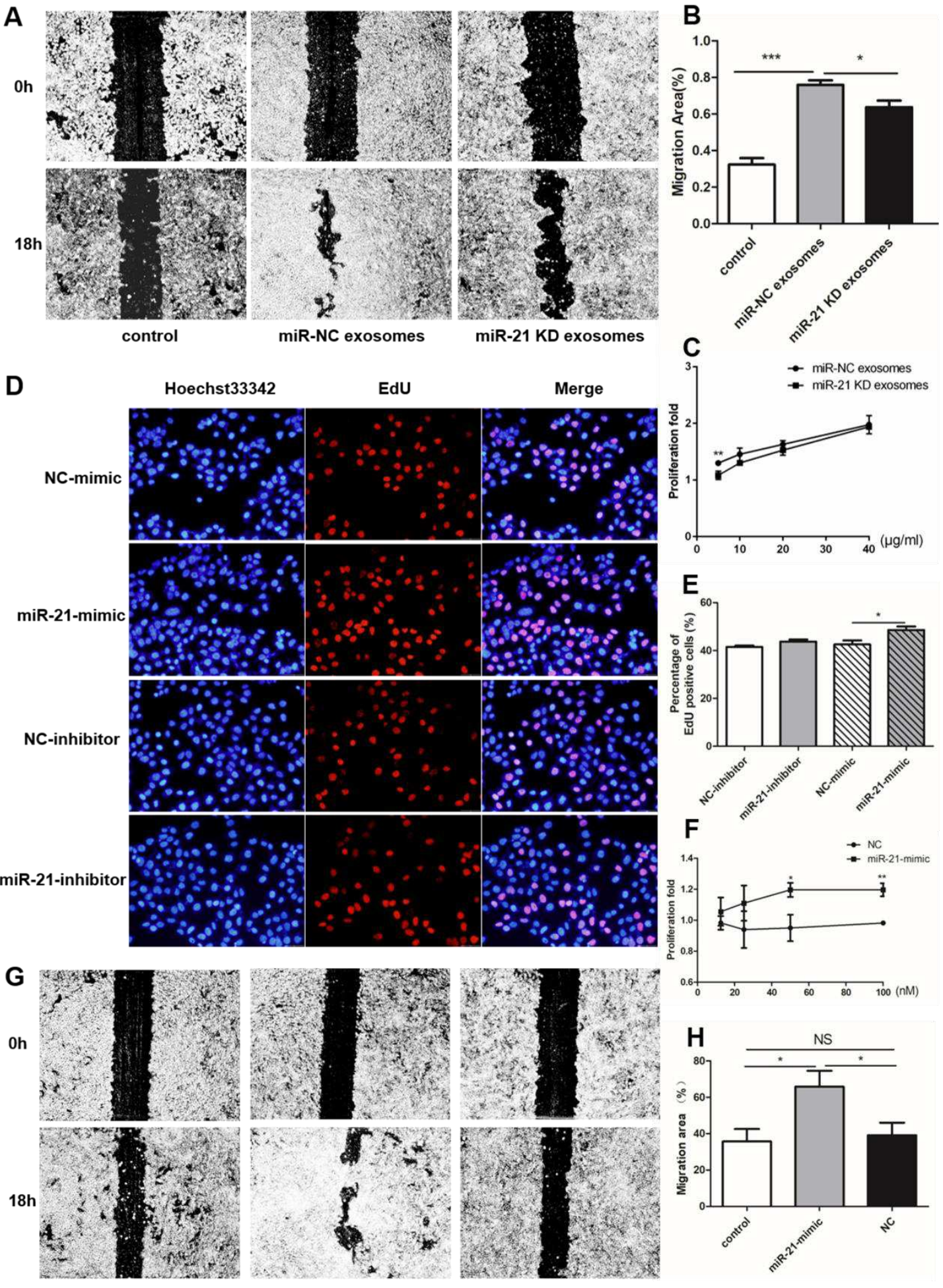

Fgure. 7

miR-21 involved in the process of HUMSC-exosomes promote cell proliferation and migration. (a, b) HCECs were 


\section{Schematic diagram}

Figure. 8

exosomes treated group was lower than the miR-21 contained HUMSC-exosomes treated group after 18 hours, $n=3$.

(d, e) The proliferation of HCECs was detected by EdU incorporation after transfected with miR-21 mimics (at final concentration of $50 \mathrm{nM}$ ). Blue: nuclear staining (Hoechst33342); Red: EdU staining, $n=3$. (f) The CCK-8 assay showed the proliferation of the miR-21 mimics group was higher than control group after 48 hours, $n=3$. (g, h) The scratch assay showed significantly faster wound closure in HCECs incubated with miR-21 mimics than NC after 18 hours, $n=5$. Data are expressed as the means \pm SD. $* P<0.05$, $* * P<0.01$, and $* * * P<0.001$. KD, knockdown

MSC

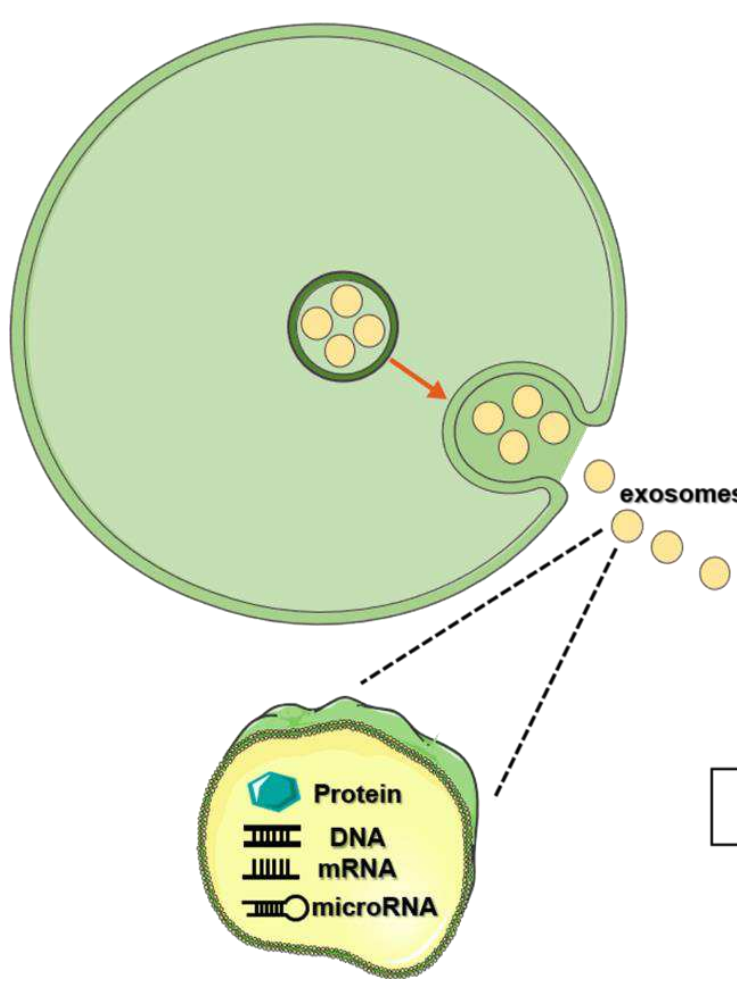

HCEC

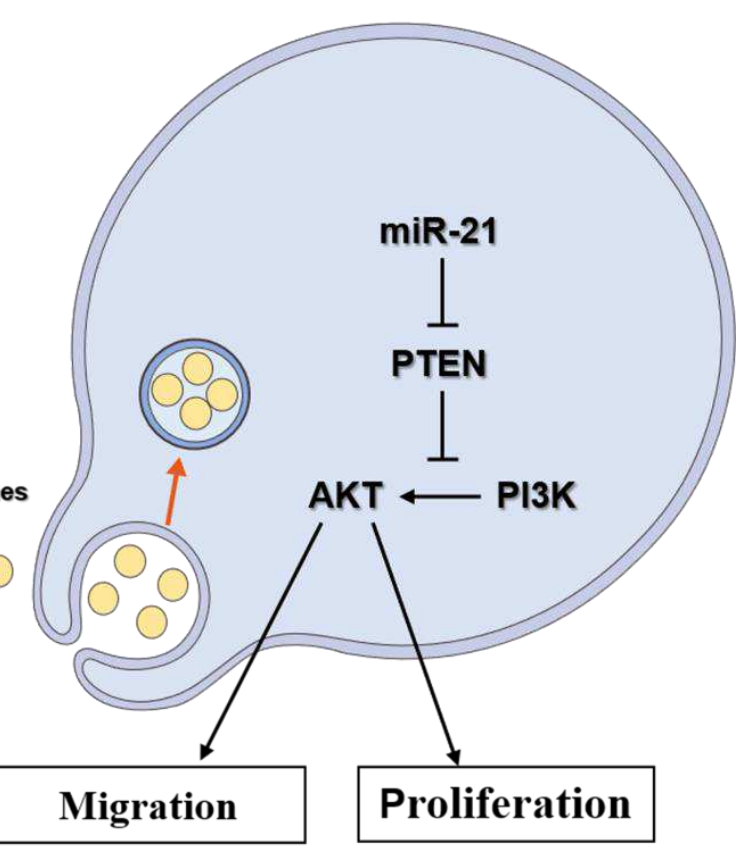

Schematic diagram describes the mechanism of HUMSC-exosomes in corneal epithelial defect. 
We first determined the positive effects of exosomes derived from HUMSCs in promoting corneal epithelial wound healing, then determined the main molecules PI3K/Akt in the wound healing process and the fact that miR-21 was the most abundantly contained in exosomes through bioinformatics analysis. Finally, we anchored PTEN as the downstream target of miR-21, which was the key link between miR-21 and related protein, and determined that PTEN/PI3K/Akt were involved in cell proliferation and migration.

\section{DISCUSSION}

Corneal epithelial damage is one of the most common ocular disorders, and novel treatments are needed to improve clinical outcomes for this type of disease. The current study demonstrated the beneficial effect of HUMSC-exosomes on corneal injury. To elucidate the potential mechanism associated with this activity, our in vitro results revealed that HUMSC-exosomes promotes HCECs proliferation and migration via repression of PTEN expression and downstream effects involving the phosphorylation of Akt. Moreover, exosomal miR-21 as an important regulator also showed the effect in promoting HCECs proliferation and migration by targeting PTEN. Our results suggested that HUMSC-exosomes may be an exceptionally meaningful and promising approach for the healing of corneal defects.

As a cell-based therapy for treating human diseases has gained increasing interest over the last few decades, hundreds of clinics or clinical trials using human MSCs have carried out, and showed that the application of MSCs can enhance wound healing[22] and ameliorate fibrosis[23]. In this study, we have shown that subconjunctival injection of HUMSCs facilitated corneal epithelial wound healing. However, several recent studies suggest that the therapeutic effects of MSCs may 
be largely mediated by paracrine effects involving proteins/peptides, hormones and vesicles packaging various molecules[24]. Among such effectors, exosomes are considered to be the key effectors to exert therapeutic function. In order to further confirm if HUMSCs paracrine effects dominate the process of cornea wound healing, HUMSCs were pretreated with GW4869, a exosomes generation blocker[25]. Expectedly, HUMSCs were less effective in enhancing wound healing when the release of exosomes was blocked. These results implicating the function of HUMSCs particularly rely on exosomes release into the microenvironment.

Researches have shown that independent application of MSC-exosomes can also play a critical role in promoting the repair of damaged tissues [26]. Moreover, MSC-exosomes have many advantages over MSCs, such as less safety concerns [27], long-term preservation and easy transportation [28], lower immunogenicity [29], capacity to cross biological barriers [30]. Previous studies have shown that exosomes from corneal MSCs can reduce scar formation and increase the transparency of corneal healing [31]. Exosomes from placental MSCs can reduce the inflammatory response during corneal alkali burn and promote the restoration of normal corneal structure [32]. Exosomes from bone marrow MSCs promoted survival of retinal ganglion cells and regeneration of their axons through miRNA-dependent mechanisms[33]. In present study, for the first time, we proved that HUMSC-exosomes could promote the repair of corneal epithelium integrity and the healing process of corneal injury both in vitro and in vivo.

Since miRNAs was first identified by Lee RC, new miRNAs are still being discovered with the development of high-throughput sequencing technologies and computational and bioinformatics 
prediction methods [34]. Increasing evidences indicated that exosomal miRNAs can prevent target mRNA from translating into protein as posttranscriptional regulation [21]. In most cases, miRNAs interact with the $3^{\prime}$ UTR of target mRNAs in a complementary manner to suppress protein translation and then regulate cell proliferation, differentiation, development and senescence $[35,36]$. Acting as the crucial mediators of MSC-exosomes, miRNAs can provide sustained therapeutic effect and fundamental alterations of the local microenvironment, making it an ideal therapeutic biomolecule [37]. Many studies have validated the role of miRNAs in exosomes in various types of cells $[38,39]$. In order to further explore how HUMSC-exosomes affects the corneal epithelial cells, we consulted GEO dataset and combined with bioinformatic analysis methods to analyze the content composition of exosomal miRNAs, and transcriptome sequencing was performed to identify the DEGs in HUMSC-exosomes treated HCECs compared to untreated condition. We found that exosomes derived from HUMSCs were rich in miR-21, which might act as the physiological and pathological regulatory factor. In our study, the exosomes extracted from miR-21 KD HUMSCs weaken the effect on HCECs proliferation and migration compared with those extracted form miR21 contained HUMSCs, implicating the function of HUMSC-exosomes partly depends on miR-21. miR-21 overexpression has the similar effect on promoting proliferation and migration of corneal epithelial cells. These results showed that miR-21 has a fundamental function on corneal epithelial cell amplification. miR-21 is a proliferation-related miRNA, and its role in wound healing was demonstrated in skin wound models [40] and cornea wound healing [41]. Despite previews results proved miR-21/SPRY2 axis participated in modulating epithelial phenotypes, promoted the migration of corneal epithelial cells and enhance the wound healing process, the mechanisms underlying miR-21 effect on corneal epithelial wound healing remain largely unknown. 
$479 \mathrm{PI} 3 \mathrm{~K} /$ Akt pathway is a signal transduction pathway closely related to cell growth and proliferation, and plays an important mediating role in proliferation, differentiation and apoptosis of normal cells.

The signal protein activity was increased in the tissue cells with strong proliferation ability [42].Studies have shown that the activation of PI3K and Akt can trigger and accelerate the transformation and proliferation of skin epithelial cells, while the use of inhibitors can inhibit the proliferation of cancer cells and improve the level of programmed cell death $[43,44]$. Once the PI3K/Akt Signaling pathway was suppressed, corneal epithelial migration was delayed [45-47]. These observations from various experiments suggest that PI3K/Akt signaling may have the stimulatory effect in the maintenance of the corneal epithelium integrity. In our experiment, PI3K/Akt pathways were activated in HCECs proliferation and migration promoted by HUMSCexosomes. miR-21 could weaken the expression level of PTEN, and increase PI3K/Akt signaling activation in HCECs.

The downstream of miR-21 has been verified based on the starBase database prediction, dualluciferase reporter gene assay and evidences form other researches [48-50]. PTEN was the potential effector, which belongs to tumor suppressor gene and inhibits the phosphorylation level of key proteins in various signaling pathways to play a negative function by promoting cell apoptosis and cell cycle arrest, and regulating cell migration and other links [51]. Recent studies have shown that PTEN is involved in the pathological mechanism of myocardial injury and neurocognition, also in regulating corneal epithelial defects [47, 52-54]. In addition, PTEN remains the main negative regulator of PI3K/Akt signaling through its phosphoinositide phosphatase activity [55]. 
500

501

502

503

504

505

506

507

508

509

510

511

512

513

514

515

516

517

518

519

520

521

To confirm the relationship among miR-21, PTEN and PI3K/Akt, we transferred miR-21mimics into HCECs, and found that miR-21 overexpression could down-regulate the expression level of PTEN, and this down-regulation further induced the up-regulation of phospho-Akt. These results demonstrated that miR-21 promoted HCECs proliferation and migration by regulating PI3K/Akt via PTEN.

Exosomes are nano-sized vesicles which could be delivered using a needle as small as possible, and their biological activity would not be affected by the increased inner pressure of the needle. We proposed that HUMSC-exosomes can not only be used as a local drug to promote corneal epithelial defects, but also can be injected for more intraocular diseases that cannot be treated locally, thus serving as a putative therapeutic agent. Although we substantiated that miR-21 in HUMSC derivedexosomes mediated the effect of proliferation and migration in HCECs, there still remains unclear whether other exosomal cargoes (protein, DNA, liquid) function as similar roles awaits further investigations. In addition, as the situation of patient with severe corneal epithelial defect is much more complex than that in animal models, whether HUMSC-exosomes can promote the healing of severe corneal injury in clinical practice remains unknown. However, our study proved that the administration of HUMSC-exosomes eye drops is a promising strategy for the treatment of corneal epithelial defect, which serves as a foundation for the development of more effective strategy in corneal wound healing.

\section{Conclusions}

In conclusion, this study firstly revealed the function of HUMSC-exosomes in promoting corneal 
522

523

524

525

526

527

528

529

530

531

532

533

534

535

536

537

538

539

540

541

542

543

544

545

epithelial cell proliferation and migration via up-regulating the PI3K/Akt signaling pathway though restraining PTEN by transferring miR-21, leading to better corneal wound repair and regeneration.

Our results offer a novel therapeutic agent for the treatment of a corneal wound as a cell-free therapy.

\section{Data Availability}

The data that support the findings of this study are available from the corresponding author upon reasonable request.

\section{Conflicts of Interest}

The authors declare no conflict of interest regarding the publication of this paper.

\section{Acknowledgments}

This work was supported by the National Natural Science Foundation of China (Grant No. U20A20363, 81970776, 8210061916), the Natural Science Foundation of Heilongjiang Province, China (Grant No. LH2020H039).

\section{Authors' Contributions}

Xiaolong Liu and Xuran Li contributed equally to this work.

\section{References}

1. Resnikoff, S., D. Pascolini, D. Etya'ale, I. Kocur, R. Pararajasegaram, G.P. Pokharel, and S.P. Mariotti, Global data on visual impairment in the year 2002. Bull World Health Organ, 2004. 82(11): p. 844-51.

2. Kimbrel, E.A. and R. Lanza, Next-generation stem cells - ushering in a new era of cell-based 
therapies. Nature reviews. Drug discovery, 2020. 19(7): p. 463-479.

3. Mathew, B., S. Ravindran, X. Liu, L. Torres, M. Chennakesavalu, C.-C. Huang, L. Feng, R. Zelka, J. Lopez, M. Sharma, and S. Roth, Mesenchymal stem cell-derived extracellular vesicles and retinal ischemia-reperfusion. Biomaterials, 2019. 197: p. 146-160.

4. Toma, C., W.R. Wagner, S. Bowry, A. Schwartz, and F. Villanueva, Fate of culture-expanded mesenchymal stem cells in the microvasculature: in vivo observations of cell kinetics. Circ Res, 2009. 104(3): p. 398-402.

5. Fan, X.-L., Y. Zhang, X. Li, and Q.-L. Fu, Mechanisms underlying the protective effects of mesenchymal stem cell-based therapy. Cellular and molecular life sciences: CMLS, 2020. 77(14): p. 2771-2794.

6. Heldring, N., I. Mäger, M.J. Wood, K. Le Blanc, and S.E. Andaloussi, Therapeutic Potential of Multipotent Mesenchymal Stromal Cells and Their Extracellular Vesicles. Hum Gene Ther, 2015. 26(8): p. 506-17.

7. An, Y., S. Lin, X. Tan, S. Zhu, F. Nie, Y. Zhen, L. Gu, C. Zhang, B. Wang, W. Wei, D. Li, and J. Wu, Exosomes from adipose-derived stem cells and application to skin wound healing. Cell proliferation, 2021. 54(3): p. e12993.

8. Tang, Y., Y. Zhou, and H.-J. Li, Advances in mesenchymal stem cell exosomes: a review. Stem cell research \& therapy, 2021. 12(1): p. 71.

9. Qiao, L., S. Hu, S. Liu, H. Zhang, H. Ma, K. Huang, Z. Li, T. Su, A. Vandergriff, J. Tang, T. Allen, P.U. Dinh, J. Cores, Q. Yin, Y. Li, and K. Cheng, microRNA-21-5p dysregulation in exosomes derived from heart failure patients impairs regenerative potential. The Journal of clinical investigation, 2019. 129(6): p. 2237-2250.

10. Sun, Y., H. Shi, S. Yin, C. Ji, X. Zhang, B. Zhang, P. Wu, Y. Shi, F. Mao, Y. Yan, W. Xu, and H. Qian, Human Mesenchymal Stem Cell Derived Exosomes Alleviate Type 2 Diabetes Mellitus by Reversing Peripheral Insulin Resistance and Relieving beta-Cell Destruction. ACS Nano, 2018. 12(8): p. 7613-7628.

11. Marote, A., F.G. Teixeira, B. Mendes-Pinheiro, and A.J. Salgado, MSCs-Derived Exosomes: CellSecreted Nanovesicles with Regenerative Potential. Front Pharmacol, 2016. 7: p. 231.

12. Lopez-Verrilli, M.A., A. Caviedes, A. Cabrera, S. Sandoval, U. Wyneken, and M. Khoury, Mesenchymal stem cell-derived exosomes from different sources selectively promote neuritic outgrowth. Neuroscience, 2016. 320: p. 129-39.

13. Krol, J., I. Loedige, and W. Filipowicz, The widespread regulation of microRNA biogenesis, function and decay. Nat Rev Genet, 2010. 11(9): p. 597-610.

14. Dong, H., J. Lei, L. Ding, Y. Wen, H. Ju, and X. Zhang, MicroRNA: function, detection, and bioanalysis. Chem Rev, 2013. 113(8): p. 6207-33.

15. Zhao, G., F. Liu, Z. Liu, K. Zuo, B. Wang, Y. Zhang, X. Han, A. Lian, Y. Wang, M. Liu, F. Zou, P. Li, X. Liu, M. Jin, and J.Y. Liu, MSC-derived exosomes attenuate cell death through suppressing AIF nucleus translocation and enhance cutaneous wound healing. Stem cell research \& therapy, 2020. 11(1): p. 174.

16. Fang, S., C. Xu, Y. Zhang, C. Xue, C. Yang, H. Bi, X. Qian, M. Wu, K. Ji, Y. Zhao, Y. Wang, H. Liu, and $X$. Xing, Umbilical Cord-Derived Mesenchymal Stem Cell-Derived Exosomal MicroRNAs Suppress Myofibroblast Differentiation by Inhibiting the Transforming Growth Factor-6/SMAD2 Pathway During Wound Healing. Stem cells translational medicine, 2016. 5(10): p. 1425-1439.

17. Di, G., X. Du, X. Qi, X. Zhao, H. Duan, S. Li, L. Xie, and Q. Zhou, Mesenchymal Stem Cells Promote 

Macrophage Switch. Investigative ophthalmology \& visual science, 2017. 58(10): p. 4344-4354.

18. Cao, L., E.O. Graue-Hernandez, V. Tran, B. Reid, J. Pu, M.J. Mannis, and M. Zhao, Downregulation of PTEN at corneal wound sites accelerates wound healing through increased cell migration. Investigative ophthalmology \& visual science, 2011. 52(5): p. 2272-2278.

19. Chang, F., J.T. Lee, P.M. Navolanic, L.S. Steelman, J.G. Shelton, W.L. Blalock, R.A. Franklin, and J.A. McCubrey, Involvement of PI3K/Akt pathway in cell cycle progression, apoptosis, and neoplastic transformation: a target for cancer chemotherapy. Leukemia, 2003. 17(3): p. 590603.

20. Pan, Y., X. Hui, R.L.C. Hoo, D. Ye, C.Y.C. Chan, T. Feng, Y. Wang, K.S.L. Lam, and A. Xu, Adipocytesecreted exosomal microRNA-34a inhibits M2 macrophage polarization to promote obesityinduced adipose inflammation. J Clin Invest, 2019. 129(2): p. 834-849.

21. Zhu, Z., Y. Zhang, Y. Zhang, H. Zhang, W. Liu, N. Zhang, X. Zhang, G. Zhou, L. Wu, K. Hua, and J. Ding, Exosomes derived from human umbilical cord mesenchymal stem cells accelerate growth of VK2 vaginal epithelial cells through MicroRNAs in vitro. Hum Reprod, 2019. 34(2): p. 248260.

22. Liu, W., M. Yu, D. Xie, L. Wang, C. Ye, Q. Zhu, F. Liu, and L. Yang, Melatonin-stimulated MSCderived exosomes improve diabetic wound healing through regulating macrophage $M 1$ and M2 polarization by targeting the PTEN/AKT pathway. Stem Cell Res Ther, 2020. 11(1): p. 259.

23. Yin, F., W.Y. Wang, and W.H. Jiang, Human umbilical cord mesenchymal stem cells ameliorate liver fibrosis in vitro and in vivo: From biological characteristics to therapeutic mechanisms. World J Stem Cells, 2019. 11(8): p. 548-564.

24. Xunian, Z. and R. Kalluri, Biology and therapeutic potential of mesenchymal stem cell-derived exosomes. Cancer Sci, 2020. 111(9): p. 3100-3110.

25. Zhang, Y.Z., F. Liu, C.G. Song, X.L. Cao, Y.F. Zhang, H.N. Wu, C.J. Guo, Y.Q. Li, Q.J. Zheng, M.H. Zheng, and $\mathrm{H}$. Han, Exosomes derived from human umbilical vein endothelial cells promote neural stem cell expansion while maintain their stemness in culture. Biochem Biophys Res Commun, 2018. 495(1): p. 892-898.

26. Phinney, D.G. and M.F. Pittenger, Concise Review: MSC-Derived Exosomes for Cell-Free Therapy. Stem Cells, 2017. 35(4): p. 851-858.

27. Tatsumi, K., K. Ohashi, Y. Matsubara, A. Kohori, T. Ohno, H. Kakidachi, A. Horii, K. Kanegae, R. Utoh, T. Iwata, and T. Okano, Tissue factor triggers procoagulation in transplanted mesenchymal stem cells leading to thromboembolism. Biochemical and biophysical research communications, 2013. 431(2): p. 203-209.

28. Kim, H.J. and J.S. Park, Usage of Human Mesenchymal Stem Cells in Cell-based Therapy: Advantages and Disadvantages. Dev Reprod, 2017. 21(1): p. 1-10.

29. Vizoso, F.J., N. Eiro, S. Cid, J. Schneider, and R. Perez-Fernandez, Mesenchymal Stem Cell Secretome: Toward Cell-Free Therapeutic Strategies in Regenerative Medicine. Int J Mol Sci, 2017. 18(9).

30. Das, C.K., B.C. Jena, I. Banerjee, S. Das, A. Parekh, S.K. Bhutia, and M. Mandal, Exosome as a Novel Shuttle for Delivery of Therapeutics across Biological Barriers. Molecular pharmaceutics, 2019. 16(1): p. 24-40.

31. Samaeekia, R., B. Rabiee, I. Putra, X. Shen, Y.J. Park, P. Hematti, M. Eslani, and A.R. Djalilian, Effect of Human Corneal Mesenchymal Stromal Cell-derived Exosomes on Corneal Epithelial 
Wound Healing. Investigative Opthalmology \& Visual Science, 2018. 59(12).

32. Tao, H., X. Chen, H. Cao, L. Zheng, Q. Li, K. Zhang, Z. Han, Z.C. Han, Z. Guo, Z. Li, and L. Wang, Mesenchymal Stem Cell-Derived Extracellular Vesicles for Corneal Wound Repair. Stem Cells Int, 2019. 2019: p. 5738510.

33. Mead, B. and S. Tomarev, Bone Marrow-Derived Mesenchymal Stem Cells-Derived Exosomes Promote Survival of Retinal Ganglion Cells Through miRNA-Dependent Mechanisms. Stem cells translational medicine, 2017. 6(4): p. 1273-1285.

34. Lee, R.C., R.L. Feinbaum, and V. Ambros, The C. elegans heterochronic gene lin-4 encodes small RNAs with antisense complementarity to lin-14. Cell, 1993. 75(5): p. 843-854.

35. Lee, R.C. and V. Ambros, An extensive class of small RNAs in Caenorhabditis elegans. Science, 2001. 294(5543): p. 862-4.

36. Inukai, S., Z. Pincus, A. de Lencastre, and F.J. Slack, A microRNA feedback loop regulates global microRNA abundance during aging. Rna, 2018. 24(2): p. 159-172.

37. Rupaimoole, R. and F.J. Slack, MicroRNA therapeutics: towards a new era for the management of cancer and other diseases. Nat Rev Drug Discov, 2017. 16(3): p. 203-222.

38. Nazari-Shafti, T.Z., S. Neuber, A. Garcia Duran, Z. Xu, E. Beltsios, M. Seifert, V. Falk, and C. Stamm, Human mesenchymal stromal cells and derived extracellular vesicles: Translational strategies to increase their proangiogenic potential for the treatment of cardiovascular disease. Stem Cells Transl Med, 2020.

39. Liang, Y.C., Y.P. Wu, X.D. Li, S.H. Chen, X.J. Ye, X.Y. Xue, and N. Xu, TNF- $\alpha$-induced exosomal miR$146 a$ mediates mesenchymal stem cell-dependent suppression of urethral stricture. J Cell Physiol, 2019. 234(12): p. 23243-23255.

40. Han, Z., Y. Chen, Y. Zhang, A. Wei, J. Zhou, Q. Li, and L. Guo, MiR-21/PTEN Axis Promotes Skin Wound Healing by Dendritic Cells Enhancement. J Cell Biochem, 2017. 118(10): p. 3511-3519.

41. Zhang, Y., F. Yuan, L. Liu, Z. Chen, X. Ma, Z. Lin, and J. Zou, The Role of the miR-21/SPRY2 Axis in Modulating Proangiogenic Factors, Epithelial Phenotypes, and Wound Healing in Corneal Epithelial Cells. Invest Ophthalmol Vis Sci, 2019. 60(12): p. 3854-3862.

42. Jafari, M., E. Ghadami, T. Dadkhah, and H. Akhavan-Niaki, PI3k/AKT signaling pathway: Erythropoiesis and beyond. J Cell Physiol, 2019. 234(3): p. 2373-2385.

43. Dalirfardouei, R., A. Gholoobi, M. Vahabian, E. Mahdipour, and F. Afzaljavan, Therapeutic role of extracellular vesicles derived from stem cells in cutaneous wound models: A systematic review. Life sciences, 2021. 273: p. 119271.

44. Alzahrani, A.S., PI3K/Akt/mTOR inhibitors in cancer: At the bench and bedside. Seminars in cancer biology, 2019. 59: p. 125-132.

45. Jiang, Q.W., D. Kaili, J. Freeman, C.Y. Lei, B.C. Geng, T. Tan, J.F. He, Z. Shi, J.J. Ma, Y.H. Luo, H. Chandler, and H. Zhu, Diabetes inhibits corneal epithelial cell migration and tight junction formation in mice and human via increasing ROS and impairing Akt signaling. Acta Pharmacol Sin, 2019. 40(9): p. 1205-1211.

46. Leszczynska, A., M. Kulkarni, A.V. Ljubimov, and M. Saghizadeh, Exosomes from normal and diabetic human corneolimbal keratocytes differentially regulate migration, proliferation and marker expression of limbal epithelial cells. Sci Rep, 2018. 8(1): p. 15173.

47. Li, J., X. Qi, X. Wang, W. Li, Y. Li, and Q. Zhou, PTEN Inhibition Facilitates Diabetic Corneal Epithelial Regeneration by Reactivating Akt Signaling Pathway. Transl Vis Sci Technol, 2020. 9(3): p. 5. 
48. Cao, L.Q., X.W. Yang, Y.B. Chen, D.W. Zhang, X.F. Jiang, and P. Xue, Exosomal miR-21 regulates the TETS/PTENp1/PTEN pathway to promote hepatocellular carcinoma growth. Mol Cancer, 2019. 18(1): p. 148.

49. Li, X., Y. Dai, and J. Xu, MiR-21 promotes pterygium cell proliferation through the PTEN/AKT pathway. Mol Vis, 2018. 24: p. 485-494.

50. Liu, H.Y., Y.Y. Zhang, B.L. Zhu, F.Z. Feng, H. Yan, H.Y. Zhang, and B. Zhou, miR-21 regulates the proliferation and apoptosis of ovarian cancer cells through PTEN/PI3K/AKT. Eur Rev Med Pharmacol Sci, 2019. 23(10): p. 4149-4155.

51. Worby, C.A. and J.E. Dixon, PTEN. Annu Rev Biochem, 2014. 83: p. 641-69.

52. Boosani, C.S., P. Gunasekar, and D.K. Agrawal, An update on PTEN modulators - a patent review. Expert Opin Ther Pat, 2019. 29(11): p. 881-889.

53. Sun, Y., X. Yao, Q.J. Zhang, M. Zhu, Z.P. Liu, B. Ci, Y. Xie, D. Carlson, B.A. Rothermel, Y. Sun, B. Levine, J.A. Hill, S.E. Wolf, J.P. Minei, and Q.S. Zang, Beclin-1-Dependent Autophagy Protects the Heart During Sepsis. Circulation, 2018. 138(20): p. 2247-2262.

54. Zhang, L., S. Zhang, J. Yao, F.J. Lowery, Q. Zhang, W.C. Huang, P. Li, M. Li, X. Wang, C. Zhang, H. Wang, K. Ellis, M. Cheerathodi, J.H. McCarty, D. Palmieri, J. Saunus, S. Lakhani, S. Huang, A.A. Sahin, K.D. Aldape, P.S. Steeg, and D. Yu, Microenvironment-induced PTEN loss by exosomal microRNA primes brain metastasis outgrowth. Nature, 2015. 527(7576): p. 100-104.

55. Wise, H.M., M.A. Hermida, and N.R. Leslie, Prostate cancer, PI3K, PTEN and prognosis. Clin Sci (Lond), 2017. 131(3): p. 197-210.

56. Romero-Pozuelo, J., G. Figlia, O. Kaya, A. Martin-Villalba, and A.A. Teleman, Cdk4 and Cdk6 Couple the Cell-Cycle Machinery to Cell Growth via mTORC1. Cell Rep, 2020. 31(2): p. 107504.

57. Ma, L.L., D.W. Wang, X.D. Yu, and Y.L. Zhou, Tangeretin induces cell cycle arrest and apoptosis through upregulation of PTEN expression in glioma cells. Biomed Pharmacother, 2016. 81: p. 491-496. 